\title{
Development of a conductivity-based immunosensor for sensitive detection of methamphetamine (stimulant drug) in human urine
}

\author{
Kaoru Yagiuda, Akihide Hemmi, Satoshi Ito \& Yasukazu Asano \\ DKK Corporation, 4-13-14 Kichijoji Kitamachi, Musashino-shi, Tokyo 180, Japan
}

Tel: [81] (422) 535111 Fax: [81] (422) 522042

\begin{abstract}
Yoshito Fushinuki
Scientific Investigation Research Laboratory, Kagoshima Pref. Police HQ, 13-1 Yamashita-cho, Kagoshima-shi, Kagoshima 892, Japan

\section{Chien-Yuan Chen}

Department of Agricultural Chemistry, National Taiwan University, 1, Sec. 4, Roosevelt Rd., Taipei 106, Taiwan

\section{Isao Karube}

Research Center for Advanced Science and Technology, The University of Tokyo, 4-6-1 Komaba, Meguro-ku, Tokyo 153, Japan
\end{abstract}

(Received 13 June 1994; revised 5 June 1995; accepted 15 August 1995)

\begin{abstract}
A simple immunosensor based on a conductivity method was developed for determination of methamphetamine (MA, a stimulant drug) in urine. Anti-MA antibody was immobilized onto the surface of a pair of platinum electrodes. The reaction of MA with the antibody causes a decrease in the conductivity of the anti-MA immobilized layer between the electrodes. A linear relationship was obtained between the conductivity and MA concentration in the range of $1-10 \mu \mathrm{g} / \mathrm{ml}$. The method requires the sample to be rinsed with water on the electrodes after the immunoreaction. This detection system was applied to the determination of MA in urine and proved to be a useful and a simple detection technique of MA in forensic science in comparison with a gas chromatography-mass spectrometry method.
\end{abstract}

(C) 1996 Published by Elsevier Science Limited.

Keywords: immunosensor, conductivity method, methamphetamine, human urine 


\section{INTRODUCTION}

Recently the drug problem has become more serious in Japan. Therefore, the need for a simple, sensitive and selective method to determine the presence of the stimulant drug methamphetamine $\left(\mathrm{C}_{10} \mathrm{H}_{15} \mathrm{~N}\right.$, molecular weight $149 \cdot 24$, abbreviated as MA) in a drug abuser's urine in order to cut off the supply routes of addictive MA and to arrest MA abusers quickly has arisen. The method based on gas chromatography-mass spectrometry (GC-MS) has been used conventionally. This method is accurate, but it needs expensive instrumentation and is time-consuming. Methods based on the chemical reactivity of secondary amino groups of MA have also been used for screening MA in human urine (Makahara \& Sekine, 1984), but the selectivity for MA of these methods is low. Recently, immunoassays of MA employing polyclonal or monoclonal antibodies against MA, such as enzyme-linked immunosorbent assay (Aoki \& Kuroiwa, 1983), latex agglutination assay (Aoki \& Kuroiwa, 1985; Uda, 1991), and a piezoelectric crystal immunosensor (Miura et al., 1991) and analytical membrane (Kawashima \& Fusinuki, 1988) have been reported. However, most of these methods are time-consuming, complicated, need several operations, and are not suitable for field use. Therefore, a simpler and easy to handle portable MA checker which can be used to investigate suspects in action, is needed as a means to resolve drug problems in the drug enforcement division in Japan.

The requirements for the MA checker include portability, disposability of the sensing probe, battery operated, high selectivity, low cost, wide use of the principle, simple procedure, long-term stability and so on for personal use. In the case of the drug checker, reliability and stability of the checker are particularly important because results obtained from the checker have the possibility of affecting the judgment of a suspect and the suspect's human rights. From several preliminary experiments, we noticed excellent selectivity of immuno-reaction and good stability of conductivity detection aimed at developing a new MA sensor. A stable blank signal is one of the important factors for development of a simple detector for MA because the MA checker is often used without calibration due to the need for a quick search in the field. The stability of the blank signal affects the signal reliability which is desired in the measurement of MA. Based on this point, the conductivity method satisfies the requirements for a simple MA detector, because the conductivity of the blank is stable and repeatable.

In order to establish a practically applicable measuring system for MA, we have developed a simple immunosensor system based on the conductivity method using an antibody-immobilized electrode. The conductivity change due to a specific immuno-reaction on the electrode was utilized. This system was applied to determine proteins and stimulants such as mouse immunoglobulin G (IgG) and MA. In this study, a detection system for MA using a specific immunoreaction and a stable conductivity detector was constructed.[7] (PCT Patent pending).

\section{EXPERIMENTAL}

\section{Reagents}

\section{MA antibody}

Anti-MA antibody was prepared from rabbits. MA modified with bovin serum albumin (BSA) was synthesized according to a well-known method (Fatori \& Hunter, 1980). The solution containing $3 \mathrm{mg} / \mathrm{l} \mathrm{MA}$ modified with BSA was injected into the blood of such rabbits, 2 times a week for the first 2 months and once every 2 months for the next 6 months. MA antibody was produced in the bodies of rabbits over 8 months. The antibody was separated and purified by gel filtration after collecting blood from the rabbits. Anti-MA antibody was in $30-40 \%$ yield from $0.5 \mathrm{~g}$ of MA. The antibody was stored in a freezer.

\section{Other reagents}

Other reagents were commercially available analytical reagents or laboratory grade and were used as received. Pure water was used throughout the work.

\section{Apparatus}

The immuno-sensing system was constructed from a conductivity cell with immobilized antibody electrode and a conductivity meter (AOL-40, DKK Co., Tokyo, Japan). The structure of the conductivity cell is shown in Fig. 1. The conductivity cell consisted of a pair of platinum 


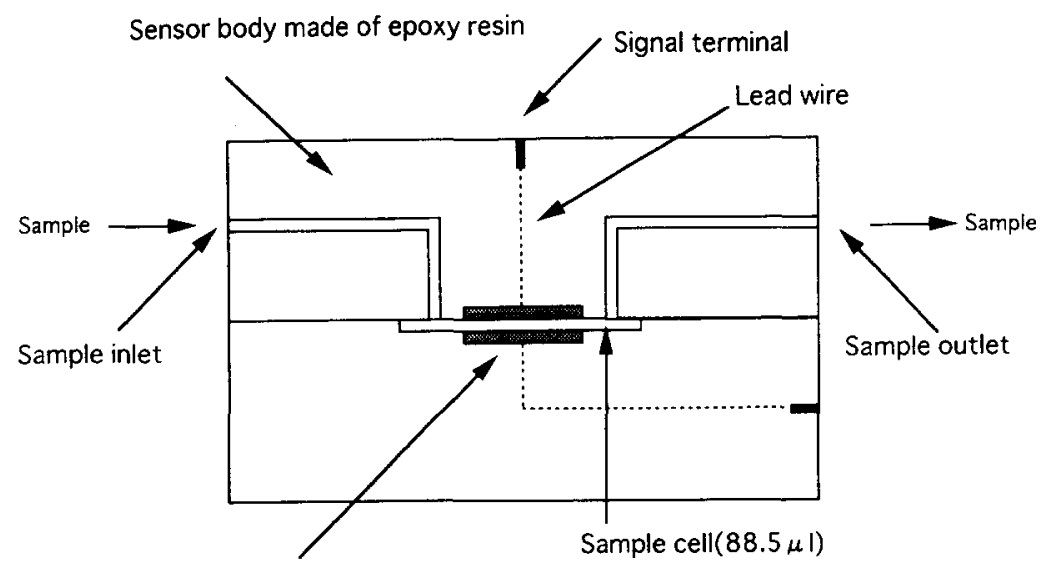

Antibody immobilized platinum electrode

Fig. 1. Structure of conductivity cell.

electrodes (surface area, $0.95 \mathrm{~cm}^{2}$ ), separated from each other by $0.5 \mathrm{~mm}$. The cell constant and cell volume were $0.05 \mathrm{~cm}^{-1}$ and $0.09 \mathrm{~cm}^{3}$, respectively. Anti-MA antibody was immobilized onto the surface of the platinum electrodes using 3-aminopropyltriethoxysilane ( $\gamma$-APTES) and glutaraldehyde. Conductivities were measured using the conductivity meter.

\section{Procedure for determination of MA}

Anti-M antibody was immobilized onto the surface of the platinum electrodes by spreading $10 \mu \mathrm{l}$ of $0.2 \% \gamma$-APTES acetone solution onto the electrodes, and incubating for $12 \mathrm{~h}$ at $120^{\circ} \mathrm{C}$. The electrodes coated with $\gamma$-APTES were rinsed with water and incubated in a $5 \%$ glutaraldehyde solution for $2 \mathrm{~h}$ at room temperature. Subsequently, the modified electrodes were incubated in an anti-MA antibody. The resulting conductivity cell with the immobilized antibody electrodes was kept at $4^{\circ} \mathrm{C}$ in a phosphate buffer solution ( $\mathrm{pH} 7.4$ ).

A procedure for detection of the immunoreaction is as follows. MA standard samples were prepared by diluting the standard solution with the phosphate buffer solution. A $100 \mu$ l sample of the MA solution was injected into the conductivity cell and incubated for $15 \mathrm{~min}$ at $25^{\circ} \mathrm{C}$. After the reaction the cell was washed and filled with pure water, then the conductivity of pure water was measured by using the conductivity detector.

\section{RESULTS AND DISCUSSION}

\section{Calibration curve}

MA concentration was measured from the conductivity change of the immuno-sensing system in pure water after the immuno-reaction. The conductivity decreased in this system. Figure 2 shows the relationship between the conductivity and the MA concentration. A linear relationship was obtained between the conductivity and the MA concentration in the range $1-10 \mu \mathrm{g} / \mathrm{ml}$. The minimum detectable concentration for MA was $0.5 \mu \mathrm{g} / \mathrm{ml}$. The conductivity was reproducible

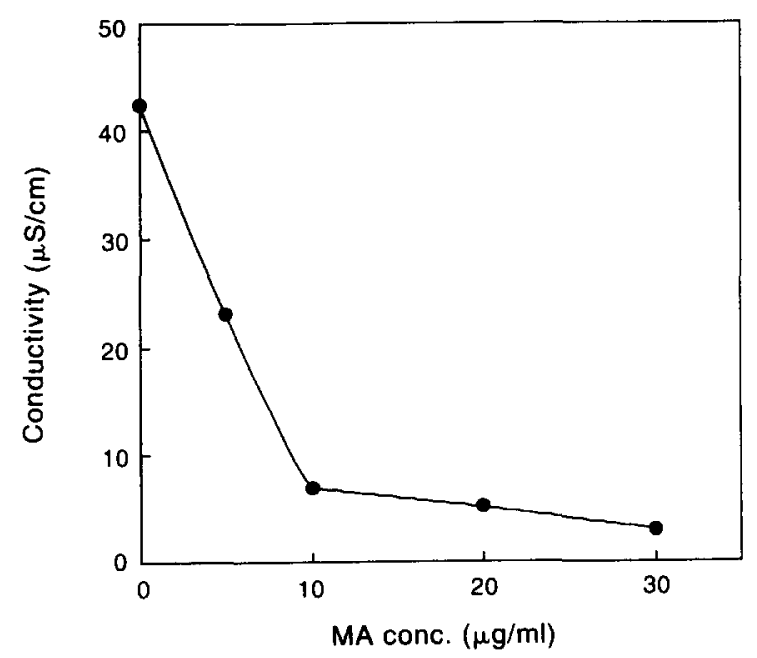

Fig. 2. Calibration curve for methamphetamine. 
within an average relative error of $5 \%$ for five time measurements of the sample containing $10 \mu \mathrm{g} / \mathrm{ml}$ MA.

On the other hand, the conductivity increased in proportion to the concentration of $\mathrm{IgG}$ as shown in Fig. 3 in case of the immobilized IgGantibody sensor. These two phenomena seem to be based on the immuno-reaction. The ionic property of the substances on the electrodes seems to be caused by the difference in the surface charge between both immunoreactions.

\section{Selectivity}

The selectivity of the conductivity detector for MA was investigated for several drugs. The concentration of the drugs was adjusted to $10 \mu \mathrm{g} / \mathrm{ml}$. No conductivity decrease was observed
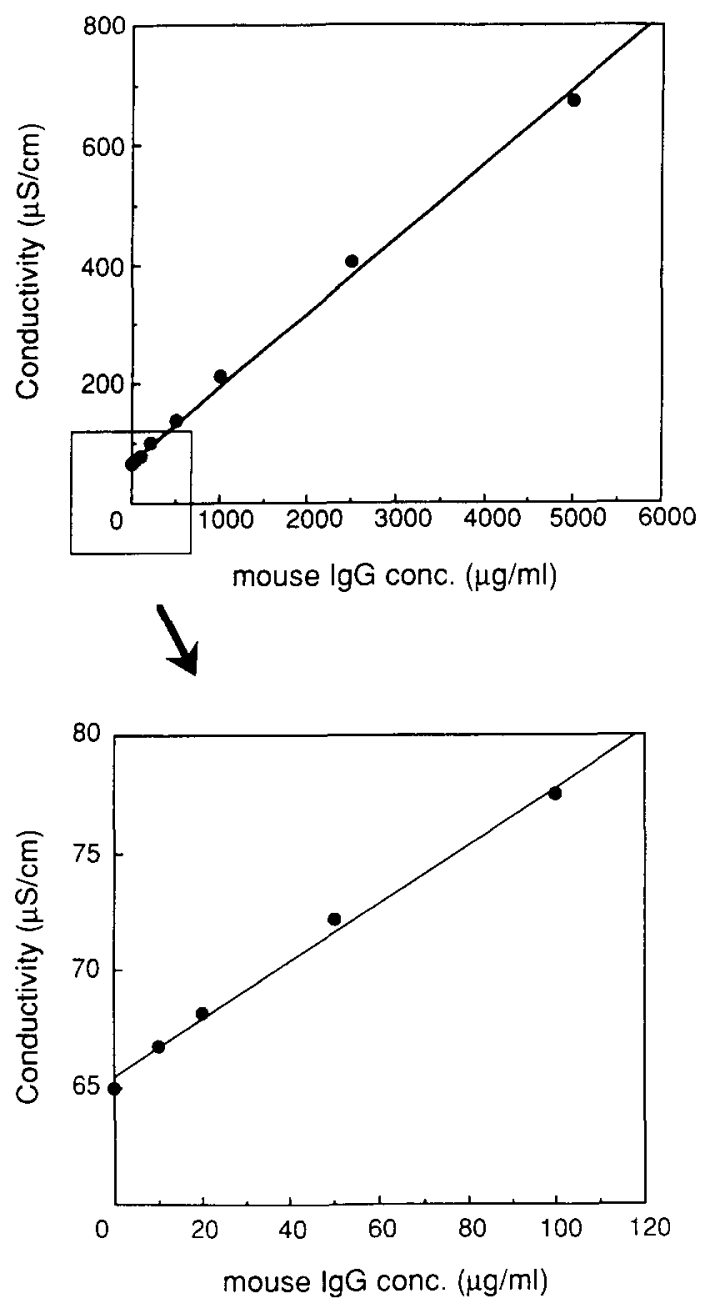

Fig. 3. Calibration curve for mouse $I g G$ for the drugs examined except for MA. The conductivity decreased only in the presence of MA. This result suggests that the specific antigen-antibody reaction can provide selective determination of MA.

The selectivity of the immunosensor developed was examined by applying it to human urine even when other components were present in the samples. The conductivity was decreased when MA was present in the sample. On the other hand, in the solution without MA, the conductivity did not decrease. We also investigated the effects of BSA, casein, HPR and starch (each component was present at a concentration of $1 \mathrm{mg} / \mathrm{ml}$ ) on the sensor signal. Little change in the conductivity was observed.

These results indicate that the developed immunosensor was applicable to the selective determination of MA in human urine.

Correlation between the present method and the conventional method

The concentration of MA in human urine of an MA abuser has generally been reported to be in the range $1-50 \mu \mathrm{g} / \mathrm{ml}$ (Kanda et al., 1978). We applied the immuno-conductivity method to the human urine sample and compared the results with those obtained by conventional GC-MS. The measurements were done using multiple sensors. We compared the samples of more than $10 \mu \mathrm{g} / \mathrm{ml}$ MA after diluting them 5-10 times with pure water. As a result, the correlation factor and regression line between both methods were $\gamma^{2}=0.994$ and $Y=1.18 X-0.52$, respectively, as shown in Fig. 4.

The sensor's measurements on eight samples had relative errors of 20 to $-50 \%$ from the standard GC-MS data. It seems that different sensors were used to determine MA for the samples.

\section{CONCLUSION}

We have developed an immunosensor for MA based on a conductivity method which detects MA in human urine from a decrease in the conductivity. The sensor has a pair of platinum electrodes with immobilized anti-MA antibody. The conductivity of the immobilized antibody layer decreases after contacting with the sample solution containing MA due to the immuno- 


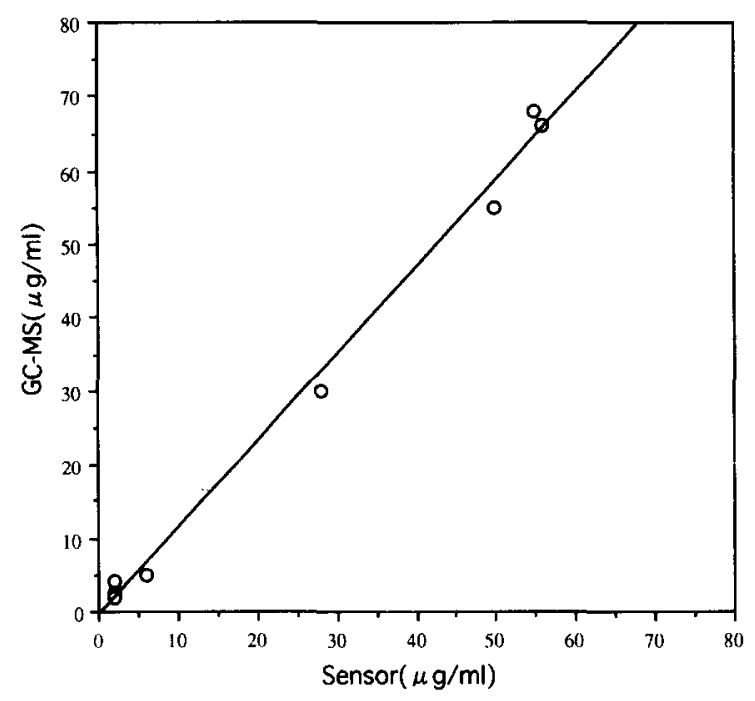

Fig. 4. Correlation between sensor method and GCMS method.

reaction. Based on this principle, determination of $\mathrm{MA}$ in the concentration range $1-10 \mu \mathrm{g} / \mathrm{ml}$ was possible within about $15 \mathrm{~min}$ for each sample. The sensor has been proven to be sensitive and stable enough to determine the critical concentration $(1 \mu \mathrm{g} / \mathrm{ml})$ of MA in human urine. As a result of this research, we concluded that the immuno-conductivity sensor is useful for screening and field-research of MA in disposable use. Efforts are under way to improve the sensitivity of the MA sensor to less than $1 \mu \mathrm{g} / \mathrm{ml}$.

The present idea can also be applied to detect other drugs such as cocaine, using the same method at the MA sensor which is currently under investigation.

\section{REFERENCES}

Aoki, K. \& Kuroiwa, Y. (1983). J. Pharmacobiodyn., 6, 33.

Aoki, K. \& Kuroiwa, Y. (1985). Forensic Sci. Int., 27, 49.

Fatori, D. \& Hunter, W. M. (1980). Clin. Chim. Acta, 100, 81 .

Kanda, Y., Inoue, T., Kishi, T., Niwase, T. \& Niwaguchi, T. (1978). Rep. Nat. Res. Inst. Police Sci. 31, 159.

Kawashima, T. \& Fusinuki, Y. (1988). Chem. Eng. 30, 21.

Makahara, Y. \& Sekine, H. (1984). Forensic Sci. Int., 26, 277.

Miura, N., Higobashi, H., Takeyasu, A., Uda, T. \& Yamazoe, N. (1991). Chem. Sensors, 1991, 7B, 53.

PCT Patent pending $(08 / 530,178)$.

Uda, T. (1991). In Chemical Sensor Technology, Vol. 3, p. 281. Kodansha, Tokyo. 